\title{
Caracterização e usos de argilas bentonitas e vermiculitas para adsorção de cobre (II) em soluçãa
}

\section{(Characterization and uses of bentonite and vermiculite clays for adsorption of copper (II) in solution)}

\author{
C. P. F. dos Santos, D. M. A. Melo, M. A. F. Melo, E. V. Sobrinho \\ CCET, Departamento de Química \\ Universidade Federal do Rio Grande do Norte \\ Av. Senador Salgado Filho, 3000, CP 1662, Natal, RN, 59072-970 \\ dmelo@matrix.com.br
}

\begin{abstract}
Resumo
Vários trabalhos tratam da remoção de metais por argilas e argilominerais. O processo de remoção geralmente ocorre por precipitação, troca iônica e adsorção. Neste trabalho estudou-se a adsorção de solução de $\mathrm{Cu}$ (II) em vermiculita, bentonitas sódica e cálcica, proveniente de Campina Grande-PB, tendo-se como variáveis $\mathrm{pH}$, tempo de contato e concentração do adsorvente. Os ensaios foram realizados à temperatura ambiente e as medidas de Absorção Atômica foram feitas no sobrenadante e no resíduo sólido. As argilas bentonitas cálcicas, provenientes de Campina-Grande (PB), sódica de Wyoming (Wyoming-USA) e sódicas (preparadas em laboratório) e as vermiculitas (provenientes do Piauí) foram caracterizadas antes e após o processo de adsorção de Cu (II) em solução através das técnicas, análise química, TG e DTA, área superficial (BET), espectroscopia na região de infravermelho, difração de raios X e medidas de pH. Os resultados mostram que as argilas estudadas adsorvem metais pesados através da troca iônica e que a vermiculita foi mais eficaz no processo, adsorvendo 40,9\% de cobre da solução em estudo, em relação às outras argilas envolvidas no processo.
\end{abstract}

Palavras-chave: vermiculita, argilas, adsorção, cobre (II).

\begin{abstract}
Mineral clays have been extensively used for metal removal. The process involves precipitation, ion exchange and adsorption. The present investigation assessed the adsorption of $\mathrm{Cu}$ (II) solution by natural vermiculite and both natural and synthetic bentonite. Tests were carried out at ambient temperature for the main parameters of the process, i.e., pH, contact time, and adsorbent content. Atomic absorption was also carried out both for the floater and solid residue. All raw materials used herein, i.e., sodic bentonite from Campina Grande-PB, sodic bentonite from Wyoming-USA, synthetic sodic bentonite and vermiculite from Piaui, were characterized both before and after adsorption of $\mathrm{Cu}$ (II) solution by chemical analysis, TG, DTA, BET surface area, infrared spectroscopy, X-ray diffraction, and $p H$ determination. The results showed that clay minerals adsorb heavy metals by ionic exchange. Vermiculite was the most efficient material studied adsorbing $40.9 \%$ of $\mathrm{Cu}$ solution.
\end{abstract}

Keywords: vermiculite, clays, adsorption, copper (II).

\section{INTRODUÇÃO}

Argilas são essencialmente silicatos hidratados de alumínio geralmente cristalinos denominados argilominerais, podendo conter ferro e magnésio. Podem conter ainda outros minerais, matéria orgânica e sais solúveis. Sob o ponto de vista físicoquímico, as argilas podem ser consideradas como sistemas dispersos de minerais, nos quais predominam partículas de diâmetro abaixo de $2 \mu \mathrm{m}$ [1]. A maioria dos argilominerais são subdivididos em função de suas propriedades estruturais e são essas propriedades que fornecem o desempenho desse material para as mais diversas aplicações [2].

As argilas são empregadas numa série de produtos acabados, por possuir propriedades adequadas, pela abundância e por ser de fácil manuseio. Apresentam poder adsorvente, podem ser empregadas como peneiras moleculares, como agentes descorantes e clarificantes de óleos vegetais e minerais, como suportes catalíticos, como agente de filtração, como adsorventes de óleos em água, etc. [3]. Entretanto, seu uso na adsorção depende de diversos fatores, tais como $\mathrm{pH}$, tipo e concentração do metal adsorvido, tempo de adsorção, além das características da argila a ser usada [4].

Neste trabalho, é apresentado um estudo de adsorção de $\mathrm{Cu}$ (II) sobre as argilas bentonita e vermiculita, previamente caracterizadas por análise termogravimétrica (TG), análise térmica diferencial (DTA), difração de raios X (DRX) e espectrofotometria de absorção na região do infra-vermelho (IV), com o objetivo de estudar melhor suas propriedades antes 
de usá-las como agente de adsorção, e quantificar o teor de cobre adsorvido sobre as mesmas. $\mathrm{O} \mathrm{pH}$ foi a principal variável e o tempo de contato foi de $24 \mathrm{~h}$, visando minimizar a agressão e poluição ambiental provocada pelas atividades industriais, onde o cobre é um dos agressores.

\section{MATERIAIS E MÉTODOS}

Neste trabalho foram utilizadas as bentonitas cálcica e sódica e a vermiculita. Todas passaram pelo processo de desaglomeração, de secagem em estufa a $80{ }^{\circ} \mathrm{C}$, de homogeneização e foram peneiradas a 100 mesh. A análise química foi efetuada em um espectrofotômetro de absorção atômica (Varian). A análise termogravimétrica foi realizada em um sistema TGA-7, modelo Perkim-Elmer, com fluxo de $\mathrm{N}_{2}$, com razão de aquecimento de $5{ }^{\circ} \mathrm{C} \cdot \mathrm{min}^{-1}$, na faixa de temperatura 30 $900^{\circ} \mathrm{C}$. A análise térmica diferencial foi realizada em sistema TGS-7, modelo Perkin-Elmer, com fluxo de ar, a uma razão de aquecimento de $10^{\circ} \mathrm{C} \cdot \mathrm{min}^{-1}$, na faixa de temperatura $30-1100^{\circ} \mathrm{C}$. A granulometria foi determinada de acordo com a norma ABNT, até tamanho de partícula de 100 mesh. Os difratogramas de raios $\mathrm{X}$ foram obtidos em um equipamento Philips, com radiação $\mathrm{Cu}-\mathrm{K} \alpha$, variando $2 \theta$ de 0 a $40^{\circ}$. A espectroscopia na região do infravermelho foi realizada em um equipamento BOMEM, na região $4000-400 \mathrm{~cm}^{-1}$, utilizando-se pastilhas autosuportadas diluídas em KBr. A umidade e a perda ao fogo foram determinadas por gravimetria, a matéria orgânica foi determinada pelo método do dicromato de potássio, o valor de $\mathrm{pH}$ das argilas foi obtido por potenciometria (pHmetro Micronal) e a capacidade de troca de cátions, determinada pelo método tradicional usado para análise de solos. Os ensaios de adsorção foram realizados em rotavapor, à temperatura ambiente, com tempo de contato de $24 \mathrm{~h}$, concentrações iniciais da solução aquosa de cobre e suspensão $18 \mathrm{~g} / \mathrm{L}$ e $20 \mathrm{~g} / \mathrm{L}$, respectivamente.

\section{RESULTADOS E DISCUSSÃO}

A Tabela I mostra os resultados da composição química das diferentes argilas usadas neste trabalho. Todas elas apresentam um teor de silício com boa correlação comparadas com o padrão. Entretanto, o teor de alumínio diverge de maneira significativa, principalmente para a bentonita cálcica, como já era esperado. Observa-se que a vermiculita apresenta óxidos de $\mathrm{Cu}^{2+}$ e $\mathrm{Zn}^{2+}$, embora em pequenos teores, o que não acontece com as bentonitas. $\mathrm{O}$ teor de $\mathrm{Mg}$ é bastante significativo para a vermiculita, pois é uma argila magnesiana, por excelência.

Todas as amostras foram caracterizadas por difração de raios $\mathrm{X}$ a fim de verificar as fases, bem como impurezas. A Fig. 1(a) apresenta o difratograma de raios $\mathrm{X}$ da vermiculita natural, mostrando um pico característico desta argila com distância interplanar de $13,78 \AA\left(6,42^{\circ} 2 \theta\right)$. Os picos que aparecem em $2 \theta$ $18,7^{\circ}$ e $21,2^{\circ}$, embora com baixa intensidade, também são característicos da vermiculita. Esta amostra apresenta como impurezas pequenas quantidades de quartzo $\left(24,96^{\circ} 2 \theta\right)$, e de sepiolita $\left(7,322\right.$ e $\left.31,28^{\circ} 2 \theta\right)$. As duas amostras de bentonitas (sódica e cálcica), também analisadas por DRX, apresentaram

Tabela I - Composição Química (\% em óxidos) e perda ao fogo das Argilas usadas como adsorventes de Cu (II) em solução aquosa.

[Table I - Chemical composition (oxide \%) and fire loss of the clays used as Cu(II) adsorbents in water solution]

\begin{tabular}{lcccc}
\hline Óxidos & $\begin{array}{c}\text { Bentonita Sódica } \\
\text { de Wyoming }\end{array}$ & $\begin{array}{c}\text { Bentonita } \\
\text { Sódica }\end{array}$ & $\begin{array}{c}\text { Bentonita } \\
\text { Cálcica }\end{array}$ & Vermiculita \\
\hline $\mathrm{Al}$ & 23,84 & 17,72 & 14,64 & 8,29 \\
\hline $\mathrm{Si}$ & 59,62 & 59,74 & 55,92 & 38,6 \\
\hline $\mathrm{Na}$ & 1,67 & 1,81 & 0,48 & 0,22 \\
\hline $\mathrm{K}$ & 0,67 & 0,46 & 0,55 & 2,86 \\
\hline $\mathrm{Mg}$ & 1,87 & 2,1 & 2,65 & 25,37 \\
\hline $\mathrm{Mn}$ & 0,05 & 0,05 & 0,03 & 0,07 \\
\hline $\mathrm{Zn}$ & - & - & - & 0,014 \\
\hline $\mathrm{Cu}$ & - & - & - & 0,015 \\
\hline $\mathrm{Cr}$ & 0,13 & - & 0,037 & 0,20 \\
\hline $\mathrm{Ni}$ & 0,08 & 0,06 & 0,06 & 0,19 \\
\hline $\mathrm{Ti}$ & 0,21 & 0,38 & 0,54 & 0,66 \\
\hline $\mathrm{Fe}$ & 7,21 & 5,2 & 8,12 & 8,93 \\
\hline $\mathrm{Ca}$ & 0,64 & 0,61 & 1,26 & 14,02 \\
\hline $\mathrm{Perda}$ ao Fogo & 13,82 & 9,72 & 16,02 & 100,149 \\
\hline $\mathrm{Total}(\%)$ & 102,08 & 103,69 & 100,31 & \\
\hline & & & & \\
\hline
\end{tabular}


picos característicos com distância interplanar de $14,7 \AA\left(6,0^{\circ}\right.$ $2 \theta$ ). Ainda, os difratogramas mostraram que estas amostras possuem como impureza o quartzo, representado pelos picos em $19,9^{\circ}, 21,0^{\circ}$ e $26,8^{\circ} 2 \theta$. A Fig. 1(b) ilustra este caso para a bentonita sódica. Ambas amostras de bentonita, sódica e cálcica, são bastante semelhantes à bentonita natural de Wyoming (USA), visto por DRX.
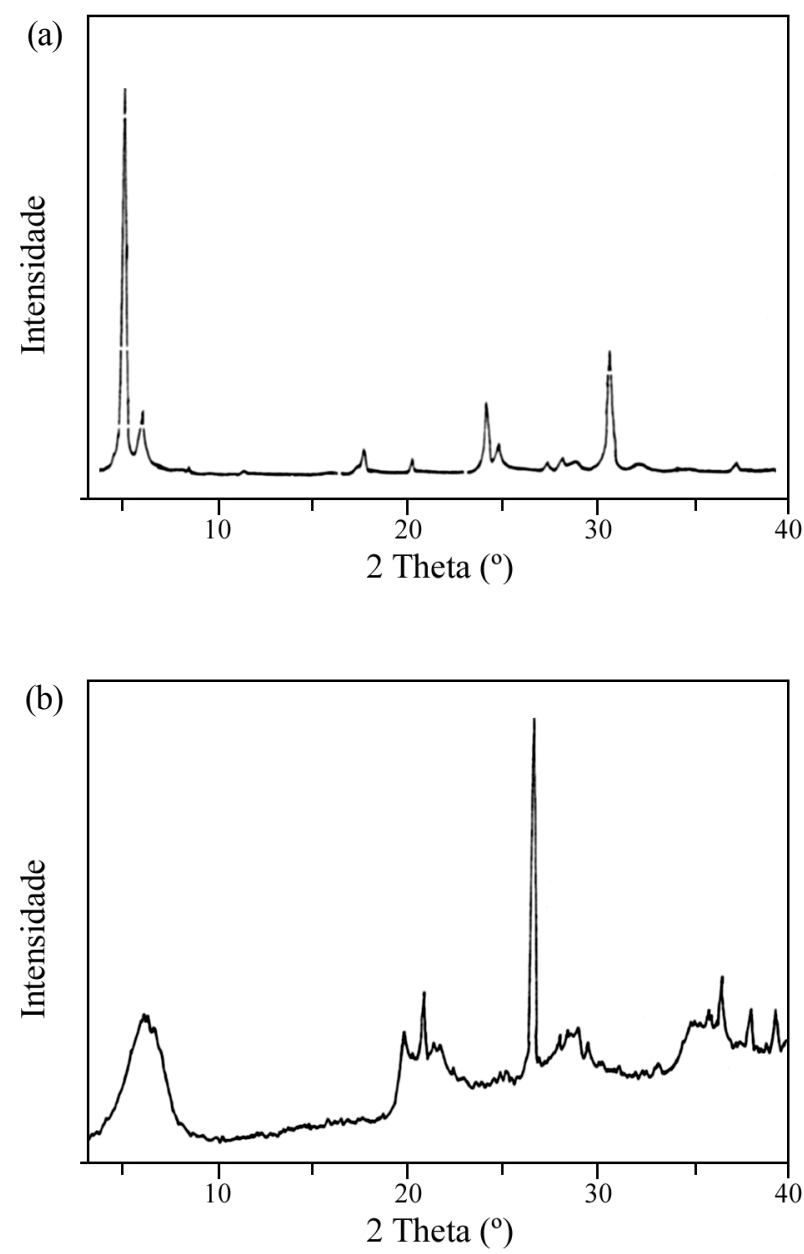

Figura 1: Difratogramas de raios $\mathrm{X}$ das amostras de argilas: (a) Vermiculita; (b) Bentonita sódica.

[Figure 1: X-ray diffraction patterns of vermiculite (a) and sodic bentonite (b).]

As amostras utilizadas nas adsorções de $\mathrm{Cu}^{2+}$ também foram caracterizadas por DRX e não apresentaram nenhuma fase diferente daquelas indicadas na Fig. 1.

A Tabela II apresenta os resultados de umidade, teor de carbono (matéria orgânica), valor de $\mathrm{pH}$ e capacidade de troca de cátions das argilas estudadas. Os resultados mostram que, embora, todas as argilas tenham sido secas previamente ao sol e em estufa, a presença da umidade é constante em todas elas e pode ser determinada de forma qualitativa e quantitativa através das primeiras perdas de massa observadas nas curvas termogravimétricas (Fig. 2).

As curvas de DTA obtidas para as bentonitas apresentam uma faixa de desidratação entre $80-210^{\circ} \mathrm{C}$, relativa às águas que
Tabela II - Umidade, teor de carbono, matéria orgânica (MO), $\mathrm{pH}$ e capacidade de troca iônica (CTC) das argilas estudadas. [Table II - Humidity, carbon content, organic matter, $\mathrm{pH}$ and ion exchange capacity of the studied clays.]

\begin{tabular}{lccccc}
\hline Argila & Umidade & $\begin{array}{c}\text { Teor de } \\
\text { carbono }\end{array}$ & $\mathrm{MO}$ & $\mathrm{pH}$ & $\begin{array}{c}\mathrm{CTC} / \\
100 \mathrm{meq}\end{array}$ \\
\hline Bentonita-Na & 4,54 & 0,4 & 0,70 & 9,3 & 42,38 \\
Wyoming & & & & & \\
\hline Bentonita-Na & 7,62 & 0,2 & 0,3 & 9,8 & 68,88 \\
\hline Bentonita-Ca & 8,5 & 0,23 & 0,4 & 9,0 & 88,28 \\
\hline Vermiculita & 8,33 & 0,48 & 0,83 & 9,4 & 99,92 \\
\hline
\end{tabular}

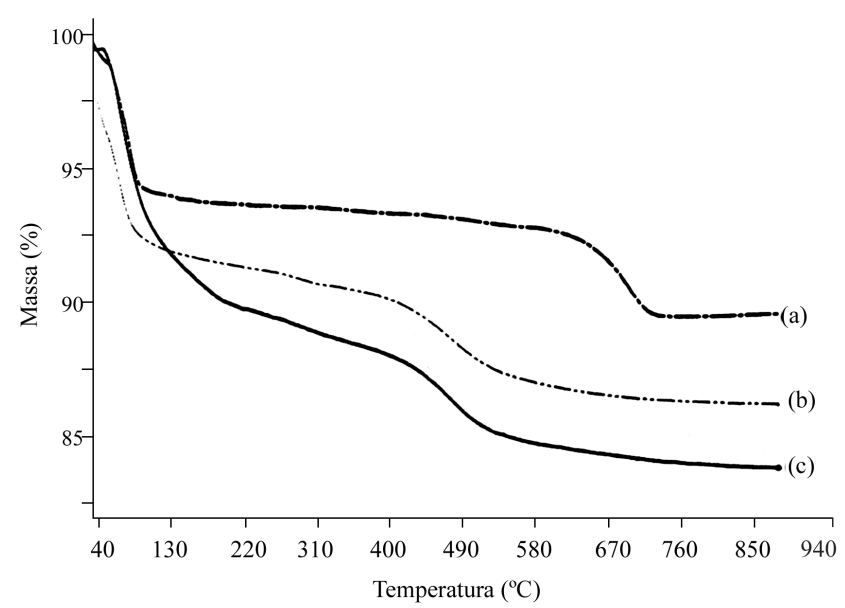

Figura 2: Curvas termogravimétricas das argilas: (a) bentonita cálcica; (b) bentonita sódica; (c) bentonita de Wyoming.

[Figure 2: Thermogravimetric analysis of $\mathrm{Ca}-(\mathrm{a}), \mathrm{Na}-(\mathrm{b})$ and Wyoming(c) bentonites.]

estão nas camadas entre as folhas de silicato, somadas às águas adsorvidas na superfície do material (umidade). A perda das hidroxilas estruturais foi observada entre 440 e $620^{\circ} \mathrm{C}$ e os picos observados acima de $800^{\circ} \mathrm{C}$ (pico duplo de caráter endoexotérmico) são atribuídos à destruição do retículo cristalino e à presença de quartzo alfa ou beta de mulita [5]. Esses resultados concordam com aqueles encontrados na literatura $[3,5]$.

Para a vermiculita, são observados três picos, todos de natureza endotérmica nas faixas de temperatura $40-110{ }^{\circ} \mathrm{C}$, $110-170{ }^{\circ} \mathrm{C} 180-270{ }^{\circ} \mathrm{C}$, atribuídos à energia de ligação das moléculas de água interlamelar ou águas de hidratação [5].

A espectroscopia na região do infravermelho das bentonitas (Fig. 3) mostra picos de estiramento assimétrico Si$\mathrm{O}$ em $1042 \mathrm{~cm}^{-1}$, vibrações de deformação Al-OH em $916 \mathrm{~cm}^{-1} \mathrm{e}$ vibrações Si-O-Al em 796 e $524 \mathrm{~cm}^{-1}$, respectivamente. As vibrações de estiramento $v_{\mathrm{O}-\mathrm{H}}$ são observadas na faixa de 3630 e $3420 \mathrm{~cm}^{-1}$ [6]. A vermiculita apresenta comportamento semelhante às bentonitas, porém a região de estiramento assimétrico é em $1004 \mathrm{~cm}^{-1}$. De certa forma, os espectros dessas argilas são bem semelhantes, principalmente no que diz respeito ao estiramento $v_{\mathrm{O}-\mathrm{H}}$, que na vermiculita está entre $3550-3450 \mathrm{~cm}^{-1}$ (Fig. 4). 


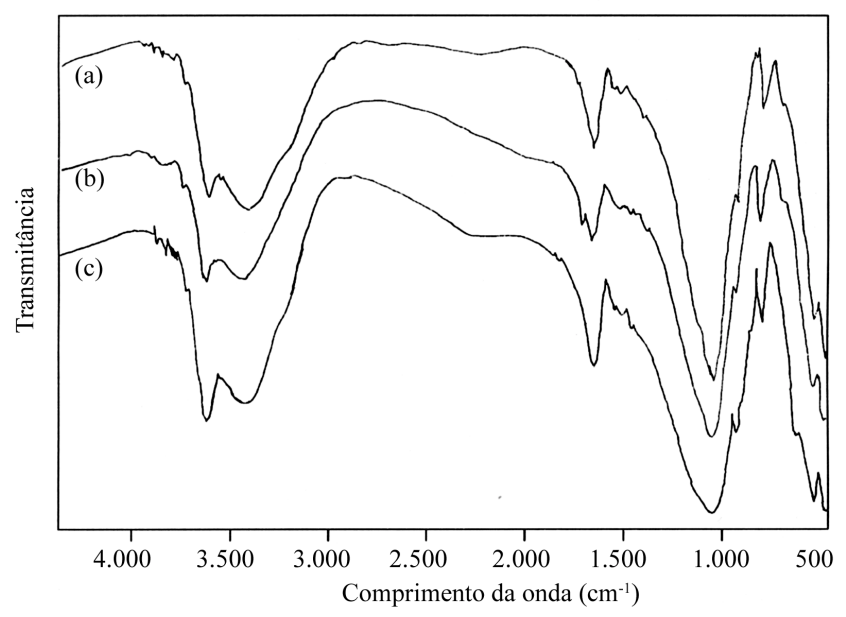

Figura 3: Espectro na região do infravermelho para as argilas: (a) bentonita cálcica; (b) bentonita sódica; (c) bentonita de Wyoming.

[Figure 3: Infrared spectra of $\mathrm{Ca}-(\mathrm{a}), \mathrm{Na}-(\mathrm{b})$ and Wyoming (c) bentonites.]

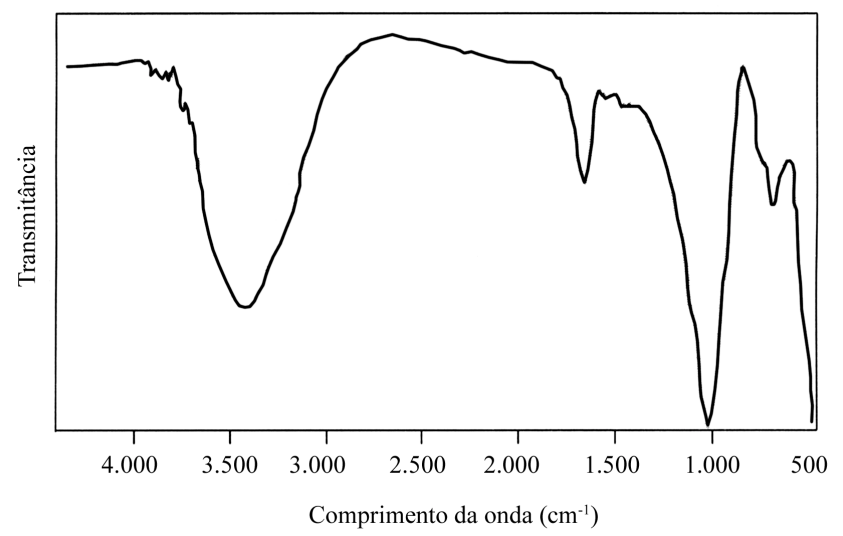

Figura 4: Espectro na região do infravermelho para a argila vermiculita natural.

[Figure 4: Infrared spectrum of natural vermiculite.]

Comparando as curvas termogravimétricas das bentonitas sódica e cálcica com aquelas adquiridas após a adsorção de cobre (II) em pH 9,5 (Figs. 5 e 6, respectivamente), verifica-se um ganho de massa, demonstrando que houve adsorção de cobre pela bentonita. Esse fato foi comprovado por absorção atômica, bem como pela coloração dos resíduos sólidos, que apresentam uma mudança de cor acentuada. Segundo Ghanem e Mikkelsen [7], quando o material é um bom adsorvente, a adsorção de cobre ocorre em poucas horas. Neste trabalho, o melhor tempo de adsorção para as argilas bentonitas sódica e cálcica e para a vermiculita foi de $24 \mathrm{~h} \mathrm{em} \mathrm{pH} 9,5$. Observou-se que a área superficial, o tamanho da partícula e a CTC influenciam a remoção do cobre pelas argilas, além, é claro, do tempo de contato. As argilas bentonitas tem menor área superficial e menor CTC do que a vermiculita, o que justifica uma menor adsorção nas bentonitas em relação à vermiculita, mantendo-se o mesmo $\mathrm{pH}$ e o mesmo tempo de contato. A curva termogravimétrica do resíduo sólido de vermiculita com

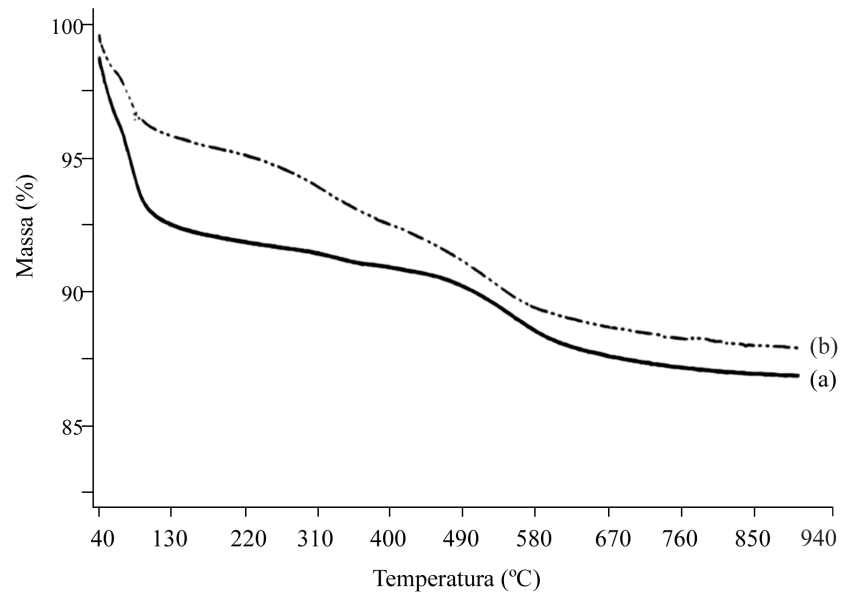

Figura 5: Curvas termogravimétricas da bentonita sódica: (a) antes e (b) depois da adsorção com $\mathrm{Cu}$ (II).

[Figure 5: Thermogravimetric analysis of Na-bentonites before (a) and after $\mathrm{Cu}(I I)$ adsorption.]

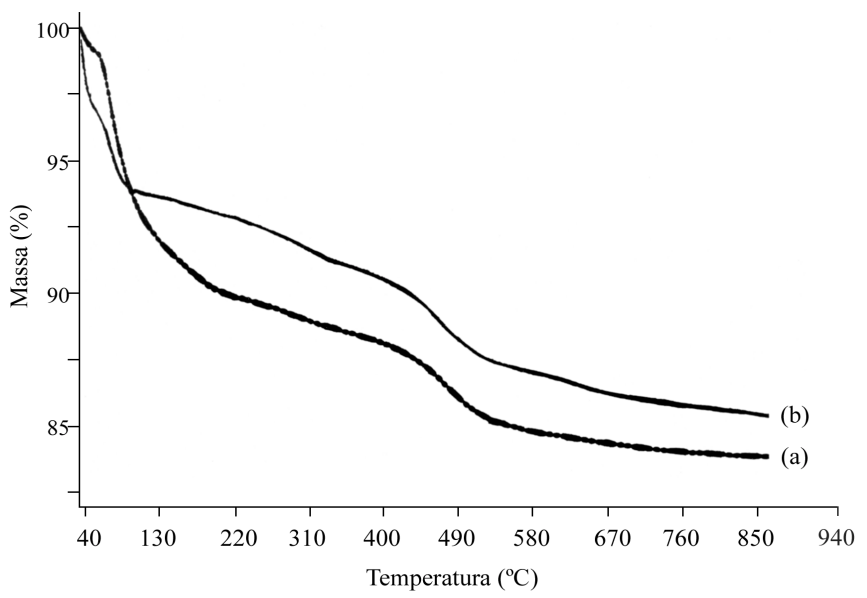

Figura 6: Curvas termogravimétricas da bentonita cálcica: (a) antes e (b) depois da adsorção com $\mathrm{Cu}$ (II).

[Figure 6: Thermogravimetric analysis of $\mathrm{Ca}$ - bentonites before (a) and after (b) $\mathrm{Cu}(\mathrm{II})$ adsorption.]

cobre (II) mostra um ganho de massa em relação à vermiculita natural, o que é coerente com os dados obtidos por absorção atômica, nas mesmas condições realizadas para as bentonitas. Esse comportamento pode ser observado na Fig. 7.

$\mathrm{O}$ efeito do $\mathrm{pH}$ foi um dos parâmetros estudados e mostra que para $\mathrm{pH} 9,5$, a remoção de cobre (II) pela vermiculita foi cerca de $40,9 \%$, para a bentonita sódica foi cerca de $30 \%$ e a bentonita cálcica apresentou uma remoção de $32 \%$ do teor de cobre(II), conforme Fig. 8. Alguns trabalhos mostram que em $\mathrm{pH} 4,0$ somente $24 \%$ do total de cobre (II) é adsorvido e em $\mathrm{pH}$ 5 cerca de $75 \%$ de cobre é adsorvido na vermiculita $[7,8]$. O trabalho em referência foi monitorado com $\mathrm{NaOH}$, o que leva a uma precipitação do cobre como espécies hidróxidos coordenados, $\mathrm{Cu}(\mathrm{OH})^{+}$, mostrando que o resultado obtido pelos autores é diferente dos resultados quando se monitora o $\mathrm{pH}$ com hidróxido de amônio, como foi feito neste trabalho. Uma das razões para a escolha do hidróxido de amônio é que o $\mathrm{Cu}$ (II) não precipita nas faixas de $\mathrm{pH}$ entre 1,5 e 4,5, o que 


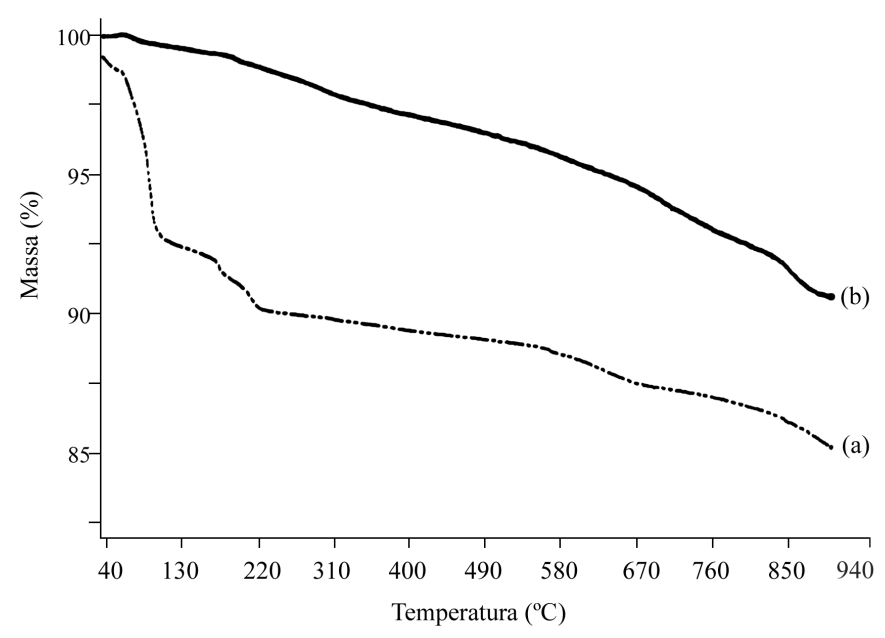

Figura 7: Curvas termogravimétricas da vermiculita (a) antes e (b) depois da adsorção com $\mathrm{Cu}$ (II).

[Figure 7: Thermogravimetric analysis of vermiculite before (a) and after (b) Cu(II) adsorption.]

poderia mascarar os resultados, porém precipita na faixa maior que 4,5 e menor que 9,3. Em pH maior do que 9,3 observa-se uma total solubilização do precipitado e o cobre fica em solução na forma de complexo, cuja cor característica é azul-escuro. Qualitativamente, a cor do sobrenadante em $\mathrm{pH}$ 1,5 - 4,5 resultante era azul claro, característica de íons de cobre hidratados $\left[\mathrm{Cu}\left(\mathrm{H}_{2} \mathrm{O}\right)_{4}\right]^{2+}$ em solução. Em pH maior do que 4,5 a solução precipita na forma de sulfato básico de cobre, cujas etapas da reação podem ser observadas nas equações $\mathrm{A}, \mathrm{B}$ e C. Na terceira etapa, observa-se a formação de íons complexos de tetramimcobre(II), de coloração azul intensa, observados na solução sobrenadante.

$$
\begin{array}{ll}
\mathrm{Cu}^{2+}+\mathrm{SO}_{4}^{2-}+\mathrm{H}_{2} \mathrm{O}+\mathrm{H}_{2} \mathrm{SO}_{4} \rightarrow \mathrm{Cu}\left(\mathrm{H}_{2} \mathrm{O}\right)_{4}{ }^{2+}+\mathrm{SO}_{4}{ }^{2-}+\mathrm{H}^{+} \\
\mathrm{pH}=1,5 & {[\mathrm{~A}]} \\
2 \mathrm{Cu}^{2+}+\mathrm{SO}_{4}{ }^{2-}+2 \mathrm{NH}_{3}+2 \mathrm{H}_{2} \mathrm{O} \rightarrow \mathrm{Cu}(\mathrm{OH})_{2} \cdot \mathrm{CuSO}_{4} \downarrow+2 \mathrm{NH}_{4}^{+}
\end{array}
$$$$
\mathrm{pH}>4,5 \quad \text { [B] }
$$$$
\mathrm{Cu}(\mathrm{OH})_{2 .} \mathrm{CuSO}_{4} \downarrow+8 \mathrm{NH}_{3} \rightarrow 2\left[\mathrm{Cu}\left(\mathrm{NH}_{3}\right)_{4}\right]^{2+}+\mathrm{SO}_{4}{ }^{2-}+2 \mathrm{OH}^{-}
$$$$
\mathrm{pH}>9,3 \quad \text { [C] }
$$

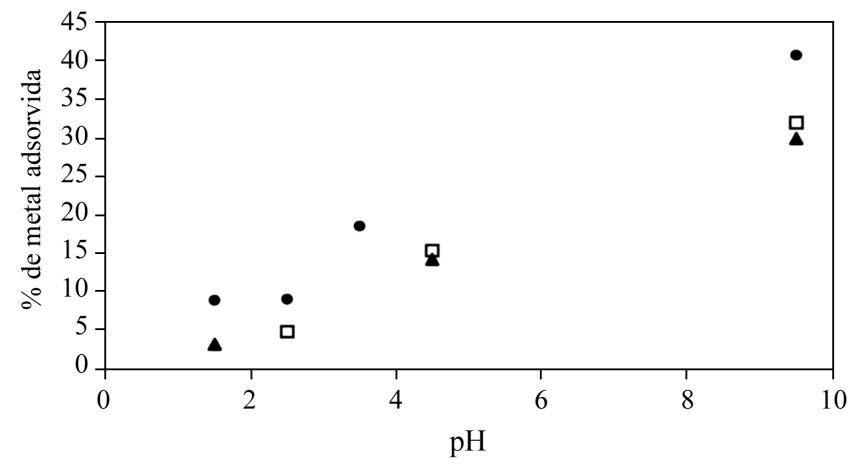

Figura 8: Influência do pH na eficiência (\%) de adsorção de cobre (II) em: $(\bullet)$ vermiculita; $(\diamond)$ bentonita cálcica; $(\square)$ bentonita sódica. [Figure 8: Influence of pH on the efficiency of adsorption of Cu(II) by vermiculite $(\bullet)$, Ca-bentonite $(\diamond)$ and Na-bentonite $(\square)$.]

\section{CONCLUSÕES}

Todas as argilas estudadas neste trabalho podem ser usadas como adsorventes de $\mathrm{Cu}$ (II), removendo-o de soluções ou águas provenientes de industrias, onde esse metal seja um agente poluente;

A vermiculita mostrou-se mais eficiente na remoção de metais por adsorção, uma vez que possui maior área específica e maior capacidade de troca iônica, favorecendo este comportamento;

A remoção do cobre pode ser considerada como um processo de troca iônica dependente da faixa de $\mathrm{pH}$ do meio;

A possibilidade de precipitação de espécies de $\mathrm{Cu}$ (II) na faixa de $\mathrm{pH}$ entre 4,5 e 9,0 foi observada com muita clareza, dificultando uma interpretação correta dos resultados.

\section{REFERÊNCIAS}

[1] R. E. Grim, Clay Mineralogy, McGraw Hill, 1 ${ }^{\text {a }}$ Ed., N.York (1953).

[2] R. E. Grim, Propriedades das Argilas: Comportamento Térmico, Cerâmica 35 (1963) 1.

[3] J. R. Souza, M. A. Melo, M. J. Dantas, D. M. Araújo Melo, "Clarificação de Efluentes da Indústria Têxtil utilizando Vermiculita Ativada como Adsorvente: Estudo Cinético e Termodinâmico", Anais do II Encontro Brasileiro sobre Adsorção 1 (2000) 365.

[4] D. M. Araújo Melo, J. A. C. Ruiz, M. A. F. Melo, E. V. Sobrinho and M. Schmal, "Preparation and characterization of terbium palygorskite clay as acid catalyst", Microporous and Mesoporous Materials 38 (2000) 345

[5] C. H. C. Pinto, "Vermiculita hidrofobizada como agente adsorvente de óleo em águas", Dissertação de Mestrado, Departamento de Engenharia Química, CT-UFRN (1994).

[6] P. S. Santos, Ciência e Tecnologia de Argilas, Ed. Edgar Blücher, 2a Ed., S. Paulo, Brasil (1989).

[7] EMBRAPA, Métodos de Análise de Solos (1979).

[8] N. C. Das, M. Bandyopadhyay, "Removal of copper (II) using vermiculite", Water Environment Research 64, 7 (1992) 852.

[9] R. S. Mackenzie, The differential thermal investigation of clays, Mineralogical Society Eds., Londres (1957).

[10] C. F. Gomes, “Argilas - O que são e para que servem”, Calouste Gulbenkian Eds., Lisboa (1986).

[11] S. A. Ghanem, D. S. Mikkelsen, "Sorption of zinc on iron hydrous oxide", Soil Sci. 146 (1988) 15.

[12] R. Yong, Y. Phadungchewit, "PH influence on selectivity and retention of heavy metals in some clay solids", J. Can. Geotech. 30 (1993) 821.

(Rec. 01/06/00, Rev. 28/10/01, Ac. 14/12/01) 